\title{
Hacking, Making, and Prototyping for Social Change
}

\author{
Ingrid Mulder and Péter Kun
}

\begin{abstract}
Even though emerging city-makers are increasingly organized to trigger social changes, it is still hard to apprehend their real power to transform space and the way we live together. In this chapter, we explore how designerly approaches, such as hacking, making, and prototyping, can empower emerging city-makers to trigger a broader change and transformation process. It can be concluded that hackable citymaking can make a difference when combining top-down public management with bottom-up social innovation. A patchable plug-in platform might enable emerging city-makers to create value for the city and for society. However, it asks for new ways of participatory governance that enable these emerging, heterogeneous city-makers to participate actively in exploring the collaborative envisioned potential and to have constructive dialogues aiming for transformational change for the common good.
\end{abstract}

Keywords City-making $\cdot$ Urban interaction design $\cdot$ Societal challenges Systemic change

\section{Introduction}

Every day, new individuals, new creative communities, and new collaborative networks get organized to 'reclaim' public space, spatially, physically, and politically. Even though they intend to trigger substantial changes, it is still hard to apprehend their real power to transform space and the way we live together. At the same time, mundane cities are being laced with sensors and mobile technologies that are generating a myriad of opportunities for developing smart solutions and generating new directions for social innovation. When these emergent technologies go urban and

I. Mulder $(\varangle) \cdot$ P. Kun

ID-Studiolab, Faculty of Industrial Design Engineering, Delft University of Technology, Delft,

The Netherlands

e-mail: mulderi@acm.org

P. Kun

e-mail: p.kun@tudelft.nl 
become embedded into our everyday lived environments, these technologies have the potential to transform our public spaces, and more importantly how we live and interact together. In the cities of the future, a digital landscape overlays our physical world. Differently put, computing is not just with us; it surrounds us, and it uses the context of our environment to empower us in more natural, yet powerful ways (Urban IxD Manifesto 2014). Urban interaction design is a forming field that explores new methodologies for new ways of city-making elaborating upon the fact that the 'making of the city' is no longer the sole concern of urban planners (Brynskov et al. 2014). No longer do their methodologies, expertise, and theories suffice to address the increasing complexity cities face. That is why designers of all sorts, IT specialists, urban anthropologists, philosophers, HCI researchers, artists, and sociologists are increasingly teaming up in coalitions that up to a few years ago were unthinkable to come up with (Brynskov et al. 2014).

Although promising, these opportunities also contribute to an increasing complexity in city-making. In the current work, we view this increasing complexity in citymaking as a collaborative design challenge and explore how designerly approaches and co-creative activities, such as hacking, making, and prototyping may bring city-making activities further than grassroots activities and generalized smart city 'visions', aiming to trigger a broader change and transformation process. In our view of city-making, smart solutions only work when they fit in with as well as arise from the everyday settings people live in.

In other words, traditional city-makers need to collaborate with grassroots' initiatives and other active citizens in changing city lives and living conditions. More specifically, we explore how the core mechanisms behind hacking, making, and prototyping intertwine, and next, we discuss how this triad can enable emerging city-makers to positively influence urban interaction design projects for systemic change.

\section{City as a Platform}

As the physical and digital aspects of a city started to interfuse and the stakeholders that create value for the city became multi-faceted, the city itself has become a patchable plug-in platform: a platform for city hacking. Platform thinking addresses the interplay of data, technology, and community. In keeping with the 'Hackable City' metaphor (de Waal et al. 2017), this interplay resembles the commercial platform of Github: a platform to connect individuals, organizations, and open-source projects to better software projects together. The city as a platform connects its citizens with its decision-makers and local projects, enabling all the stakeholders to combine topdown planning and bottom-up participation in patches to better their city.

On the city as a platform lays interaction design's stretch towards the urban scale, which also redefines how the fuzzy front-end of the design process is conducted: citizens navigating in a rich urban context, in order to improve life quality in their proximity, working with democratized technology within their reach. Aiming to get 


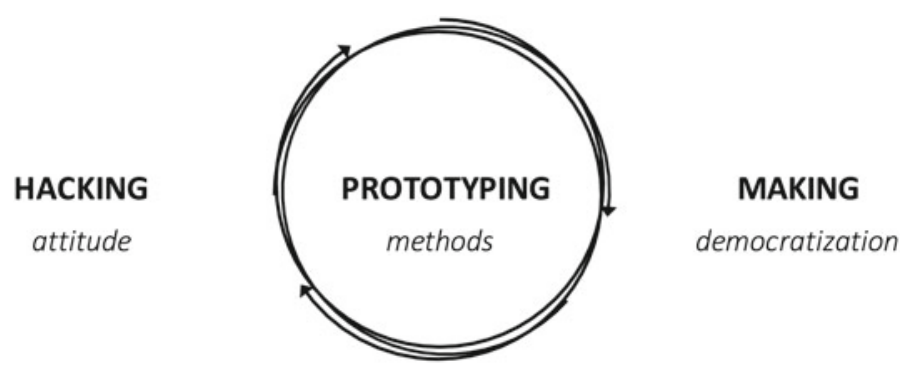

Fig. 1 Hacking, making, and prototyping in relation to each other

a more nuanced understanding of the city as a design platform, we elaborate upon the concepts hacking, making, and prototyping while entering the cityscape. In the next sections, we discuss these concepts, which hold several different connotations, and make them operational for hackable city-making, using an urban interaction design perspective and take stock of its roots in arts, technology, and activism (Fig. 1).

\subsection{Hacking}

Hacking is an ambiguous term with multiple, competing meanings in contemporary culture. Firstly, in its original meaning, hacking refers to the 'hacker culture' of software and hardware tinkerers coming from MIT and then Silicon Valley (Levy 2001). The notion that Levy calls 'true hacking' is based on the hacker ethic, with core principles, such as sharing, openness, and world improvement. These principles are referring to a general constructive behaviour. Secondly, another contemporary meaning of a hacker is associated with criminality, a person that exploits computer security systems. This notion carries negative sentiment and describes destructive behaviour that is almost the opposite of the 'true hacking' idea. Thirdly, a modern pop culture connotation of hacking is lifehacking. Lifehacking was coined by technology writer Danny O'Brien (Thompson 2005), referring to productivity and efficiency shortcuts, skills, and tricks for daily life. Such shortcuts and tricks as a hack follows the description of a kludge - a historical computer jargon term from the 1960s-as described by Koopman and Hoffman (2003: 73):

A fix that is awkward or clumsy but is at least temporarily effective or An overall design that is of questionable elegance or downright ugly.

Expanding this definition from decades ago, today the previous contemporary connotations of hacking carry a refined understanding of what a hack is: an exploratory, creative way of overcoming limitations of a system. How this takes shape is often by modifying or repurposing a certain knowledge, technique, or technology for a new use. Moreover, this repurposing also leads to a hacker attitude 'because we can', trying out if a technique works in another context. An iconic example of this thinking 
is the story of the 'Trojan Room Coffee Machine': in 1991, people at the Computer Laboratory of University of Cambridge installed a camera showing the coffee pot. The creators needed to develop many things from scratch, like server and client software, and generally went a great length for solving a non-substantial problem (Stafford-Fraser 2001). This coffee machine broadcast became the first webcam.

When looking at city-making from an urban interaction design perspective, hacking is considered as a constructive activity, such as Townsend's Civic hackers (2013) or The Hackable City project (Ampatzidou et al. 2015). In our view, hacking can indeed be seen as a social constructivist activity in city-making, and embrace a typical hacker attitude. Such an attitude can manifest as optimizing an inefficient, bureaucratic policy-making process that is often lagging behind technology advancements. An early exploration of the hacker attitude applied to city-making can be found in squatting, the movement of occupying abandoned or unoccupied buildings that the squatters do not own. There are many policies for squatting today, though a commonality is its history of operating in a legal grey zone and the corresponding legislation that was lagging behind. In the case of squatting, the hacker attitude refers to a new way of thinking around redistributing unoccupied, available buildings for residential purposes. Beyond squatting, a timely example for hacker attitude in city-making addresses the rise of various sharing economy start-ups that are operating at the borderline of governmental policies and use-abuse the lagging of policy-making to flourish. For example, Airbnb hacked the general accepted way of short-term room rental while Uber hacked the established norms in transportation services. Sharing economy start-ups often question the status quo, and in our view, this is the hacker attitude applied in city-making. Although often experienced as controversial, it is obvious that these new kinds of businesses introduced by Airbnb and Uber do not necessarily refer to a constructive hacker attitude. However, these exemplary sharing economy business cases do challenge the current, established way of city-making, and open the floor to new city-makers to trigger a systemic change for new types of social innovation. Urban interaction design operates in a world full of complexities, from stakeholders to citizen needs and policy-making; there are various layers, various actors, and various types of problems. To cut through these complexities, we argue that the hacker attitude is core: people need to be proactive to trigger change, sometimes to provoke change. Many art and design schools teach by Grace Hopper's famous quote [retrieved from CHIPS Magazine (2002)]:

It is often easier to ask for forgiveness than to ask for permission-Grace Hopper

Innovation oftentimes happens fast and is not bounded by the lagging of governmental regulations. Considering urban complexities, the core mechanisms of the hacker attitude are exploring the borders and stretching the status quo, let them be legislation or the public's thinking on a certain matter (such as ownership in the age of the sharing economy). To conclude, the hacker attitude might appear disobedient or unabashed, but also pioneering and daring to be the first, and these we find key characteristics. The hacker value for city-making lies in the potential to serve the public interest, but still too often hackers undermine the common good due to a short time, activist focus on political protest. 


\subsection{Making}

With the third industrial revolution (Rifkin 2008), manufacturing has been expected to shift towards a democratized and decentralized, distributed ecosystem, enabling the masses to realize bespoke products with modern technology, such as 3D printers, accessible electronic prototyping kits, or a blossoming open-source culture on the Internet. Pioneer activists leading this movement, the makers, initiated physical spaces all around the globe. Fablabs, Hackerspaces, and Makerspaces are appearing, providing a physical shop as well as a meeting point for like-minded people, who come together to work on their own Do-It-Yourself (DIY) projects, but also to collaborate with other makers, exchanging expertise or share knowledge. The knowledge and expertise sharing aspect of making makes it also intertwined with education all over the world, as illustrated by Mostert-van der Sar and her colleagues (2013). Educational institutions are increasingly hosting Fablabs and other fabrication workshops become available for the masses. Such connections to education provide a safe environment to test and experiment. When people visit a Fablab or a Makerspace, they are not expected to know how equipment, tools, and devices work; they go there to learn. Furthermore, specialized working tools are not available in every household, and it is hard to justify having, e.g. a soldering station or a 3D printer at home if you have never tried it beforehand. These physical spaces where making can take place are democratizing who has access to realizing various hobbies or professional projects.

Besides providing spaces for making, a vast number of tools are getting available for people that are not very tech-savvy or trained. The focus is not anymore on creating tools for experts, but to serve a broader user base including complete beginners and interested amateurs. Makers have been successful in attracting children to code, 3D print, or build in Minecraft, and this success generates demand for better end-user tools that are not overcomplicated and has the right constraints and trade-offs to still remain usable and productive after a little-learning curve. For urban interaction design this is important, because in the urban context design often happens by citizens that are not necessarily trained in design.

To leverage making in cities, the fabrication communities are essential to provide places where lead users of a city (i.e. citizens) can gather to realize technological projects that better the living in the city. In this way, making enables citizens to create bespoke solutions (patchable plug-ins) for their city; when the citizen needs are addressed by citizen-designed solutions, that is a major shift from the dominant concept of urban services being provided by the government, more often than not resulting in services that are far from how reality works. In all complexities in urban space, making democratizes the creation of bespoke solutions by providing infrastructure and knowledge and skills for the urban interaction designer. In the future, this could be further amplified by the maker community and its sharing practices on the Internet; when people upload their local bespoke products, services for peerproduction sites, such as Instructables.com. The local bespoke solutions could live a global life, getting adapted to different circumstances. 


\subsection{Prototyping}

In the past decades, the techniques and methods previously characterized with the design-related professions (e.g. industrial design, architecture) have started to find their use for a broader audience. Design thinking (Brown 2008) has gained popularity in the business world illustrating this trend of innovation and new ways of management. Living labs have grown in popularity in the past years to stimulate open, collaborative and bottom-up models of innovation where citizens are at the centre of the innovation process. A living lab can be defined as:

an experiential environment where users are immersed in a creative social space for designing and experiencing their own future. Policy makers and citizens can use living labs to design, explore, experience, and refine new policies and regulations in real-life scenarios before they are implemented. (McPhee et al. 2012: 3-4)

Where the origin of living labs started from the industry's or city management's drive for regional innovation, today's city laboratories are often initiated by emerging city-makers. Even though co-creative partnerships that join forces in developing new product and services are keys in both 'prototyping' initiatives (Mulder 2014, 2015). In the meanwhile, designers have been redefining design towards complex systems and tackling complex societal problems (Sanders and Stappers 2014). In our view, these trends illustrate that the designers' toolbox of techniques, methods, and ways of operating has the potential for cutting through the urban complexities as well.

A key aspect of problem solving (in design) is the use of prototypes. Prototypes can be all kind of artefacts, as long as they enable the different stakeholders to collaboratively explore alternatives and to articulate their different viewpoints. In this view, prototyping is a way of communication between different parties. We consider communicating via prototypes as a process where iterations happen throughout the discussion, evolving the prototypes in a trial-and-error manner towards finding the optimal design solution (Buxton 2010). Out of their context, early prototypes can easily be seen as quick hacks, and making often enables quick prototypes. In urban interaction design, prototypes are often design interventions, with the leading principle to engage the public in the conversation about the possible future. The powerful aspect of iterative development is to keep the tangible solutions close to its users, and continuously adapt the feedback in the following prototypes. These are the important aspects to abandon the principle to aim for over-polished solutions that are neverready. Like this, people can dare to envision futures with bolder ideas, iterating their way towards one prototype a time.

A manifestation of prototyping and design for solving urban or societal matters is the emergence of different design jams and hackathons-pressure cooker events that are targeted at establishing active local communities, while teaching design techniques to interested people. These types of events enrich the spectrum of the physical places connected to making and encouraging the hacker attitude to innovate solving complex problems by applying cheeky or clever thinking in repurposing of previous knowledge or techniques on new problem areas. The time frame a pressure cooker event enables is short, so people should not think too much about an approach, 
just do it and see what happens. This set-up is interventional and based on weekendlong get-togethers, consequently the outcomes might not be sustainable. However, on personal and community levels, such an approach is a boost to probe ideas, get stakeholders together, and learn new things. This transition is doing the groundwork for sustainable change for projects that has bigger potentials.

\section{Hackable City-Making: Towards Systemic Change}

Seeing the city as a platform that welcomes bottom-up social innovation and allows for hackable city-making opens the path towards system change. In keeping with Suchman et al. (2002) as well as Junginger (2008), we use prototyping as an insightgiving tool enabling society to change. Considering the complete spectrum from an idea to actual change in society, the role of hacking, making, and prototyping is ranging from prompting the idea to the creation of prototypes to communicate the idea.

We refer to the definition of social innovation as elaborated in the report of the European Policy Advisors (2010) entitled 'Empowering people, driving change: Social innovation in the European Union'. Social innovation refers to social demands that are traditionally not addressed by the market and are not directed towards or involve vulnerable groups in society. A common case of social innovation is the care for elderly, which is a ubiquitous problem in the world, and as the world's population is ageing, also addresses a growing need. Social innovation is complex from multiple aspects; the boundaries between 'social' and 'economic' blur for social challenges, which are directed towards society as a whole and often involve end-users (e.g. citizens, who proactively shape things). Further examples in cities for social innovation may be increasing social cohesion, creating sustainable living, supporting the ageing society, etc. However, in order to reshape society in the direction of a participatory arena where people are empowered, learning is central to make policies more effective. The following three approaches are interdependent and strengthen each other.

The first approach is the foundation for the second which creates the conditions for the third - an innovation that addresses a social demand (e.g. care of the elderly) contributes to addressing a societal challenge (ageing society) and, through its process dimension (e.g. the active engagement of the elderly), it contributes to reshape society in the direction of participation and empowerment. In the next section, we discuss how hackable city-making can trigger a broader change and transformation process.

To illustrate the complete life cycle of such a hackable city-making process, we adopted the six stages of social innovation as defined by Murray and his colleagues (2010) to address hackable city-making towards systemic change (see Fig. 2). Observing this model from a designerly perspective separates the 'early stages' from the 'sustaining stages' of social change. In the following part, we elaborate on this twofolded framing, to leverage the process of hacking, making, and prototyping for social change. 
Fuzzy front-end of city making

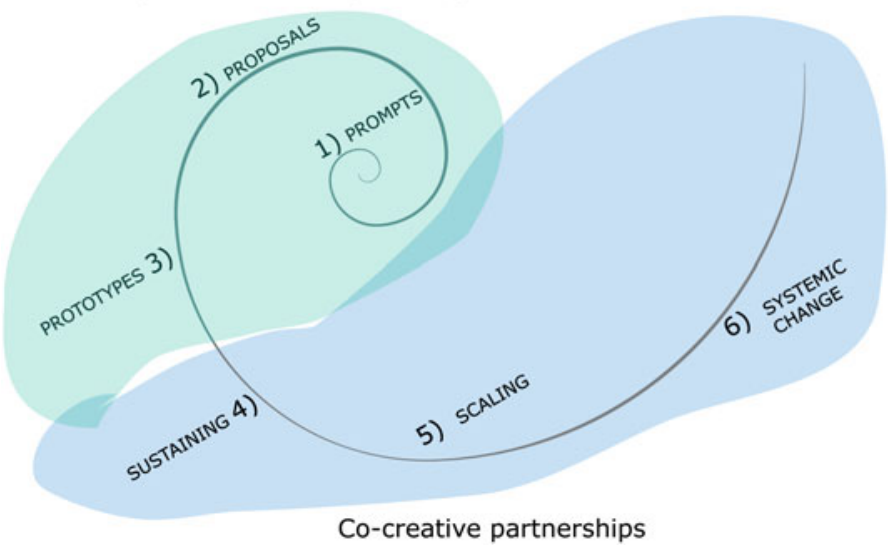

Fig. 2 Hackable city-making in relation to the six stages of social innovation

\subsection{Fuzzy Front-End of City-Making}

Following the hacking, making, and prototyping practices of doing urban interaction design, the outcomes are most frequently 'design bursts' and interventions, semi-worked out ideas; rarely take on the high-level (transformational) design challenges of establishing sustainable change. Considering Rittel and Webber's wicked problems (1973), we observe an interventional design approach that targets solving wicked problems without a typical end point:

With wicked problems [...], any solution, after being implemented will generate waves of consequences over an extended - virtually an unbounded - period of time. [...] The full consequences cannot be appraised until the waves of repercussions have completely run out, and we have no way of tracing all the waves through all the affected lives ahead of time or within a limited time span. (Rittel and Webber 1973: 163)

In an urban context, we call the 'fuzzy front-end of city-making' the first three stages of social innovation: [1 Prompts], [2 Proposals], and [3 Prototypes]. The fuzzy front-end of city-making happens often at pressure cooker events, such as hackathons or design jams. These events host people from various stakeholder groups, who are all invited to propose problems [1 Prompts, 2 Proposals], often attend workshops to work on specific problems [1 Prompts] and by DNA of hackathons create prototypes to communicate the ideas [3 Prototypes]. Reaching the [3 Prototypes] stage is relatively easy, and there are many tools available from end-user development, co-design, or making. Co-designing activities during such events (but also student projects, etc.) can flesh out preliminary/immature ideas for envisioned futures. Turning these ideas tangible is important for discussing them with the different stakeholders, but also for 'thinking through designing', to really understand the core wicked problems the idea intends to solve. All these processes have hacking, making, and/or prototyping at their core. 
As illustrated, this fuzzy front-end stage might take a long while, and the process might happen in a way that a preliminarily realized idea (e.g. as a prototype) inspired another project with another set of people, who figure a different angle to solve the problem which eventually succeed. This cross-pollination of ideas is a common characteristic of pressure cooker events, but also a cultural characteristic for social innovation and cities; cities do not exist in a vacuum, and best practices are there, out in the wild, to be copied as well as improved elsewhere.

\subsection{Co-creative Partnerships}

In keeping with Anthony Townsend (2013), we put the smart citizen in a central position. Interestingly, in a recent public debate, Townsend stressed that although hackathons are the main form to have citizens involved in demonstrating the potential of open data and smart cities, there are no best practices that stress the citizens' voices that can be scaled and sustained. Most hackathons organized nowadays act as nerdmeetups and remain stand-alone events. In our co-creative activities, we therefore deliberately elaborate upon various ways to involve 'civic hackers' and stakeholders representing a quadruple helix (van Waart et al. 2015, 2016) as well as upon the role of these co-creative activities in making them more aware of open data and the potential value in order to drive social change (Morelli et al. 2017). We refer to this quadruple helix consortium as 'co-creative partnerships' (Mulder 2014, 2015) and emphasize the human scale in a shared process of knowledge production in which they collaboratively envision desired futures (Brodersen et al. 2008; Carayannis and Campbell 2012; Mulder 2014; van Waart et al. 2016). Key to co-creative partnerships is a dialogical approach (Mulder 2014, 2015) while in most hackers' initiatives an activist approach is leading, which not necessarily lead to systemic changes that contribute to the common good.

Similarly, Manzini and Rizzo (2011) have demonstrated how 'large-scale sustainable changes' could be achieved by participatory design when citizens and designers work together, co-creative partnerships are a crucial asset to enable the collaborative activity of prototyping and scale these activities towards participatory city-making [4 Sustaining, 5 Scaling]. The fuzzy front-end activities can be interpreted as largely unobtrusive ways of building a common vision among the new city-makers for creating fertile structures and embedded areas that introduce design knowledge for the systematic exploration of new ways of city-making [6 System change].

\subsection{From Designing for to Making Together}

There is a plethora of promising cases and best practices that illustrates prompts, proposals, and/or prototypes. There might even be more good, best, and promising practices that stay unnoticed by the general publics. However, practices that address 
systemic change are hard to find. The question why it is so hard to get from the fuzzy front-end of city-making towards co-creative partnerships making a systemic change largely remains unanswered. The current debate on social design largely focuses on what design can do, showing mainly prompts and proposals that are not evidenced by impact. Similarly, many toolkits have not shown their value in sustained practice. The outcomes of a workshop to map and collect various strategies for citizens' engagement and the role of cities held at the Design \& the City conference (Amsterdam, April 22, 2016) contributed to a great bricolage of designerly approaches and a vivid discussion on how to scale up interventions and to foster system change; however, no clear guidelines could be derived (see Kun and Mulder 2016). Also, in a recent policy workshop on 'Shaping the Future of Digital Social Innovation' at the European Commission (June 29, 2016, Brussels), it was also concluded that it appears hard to sustain and scale these practices (see Mulgan 2016).

Of course, cherish and promote small experimentations is a welcome first step, but foremost the need to change designerly perspectives towards more participatory and systemic perspectives that reflect on how to activate new forms of collective action is key. The role of designers in hackable city-making moves towards orchestrators of the various stakeholders involved and includes 'designing' the relationships among them as well in order to triggering a process of broader change and transformation (Mulder and Loorbach 2016).

\section{Discussion and Conclusions}

Although promising, it is not straightforward that hacking, making, and prototyping allow for a systematic exploration of new ways of city-making. It requires a different designerly mindset, from an emphasis on the designed object towards strategic design and the process, such as the role of infrastructuring (Karasti 2014). Emerging contexts for systems perspectives in design are still in its infancy (Jones 2016). Both systems and design fields have developed largely independent and outside academia, with hardly any effort in uncovering the hidden links. Design has been largely biased by its practical materialism and driven by theories of use and human-centredness. Systems theory has been biased by its abstract nature, and lack of attention to designerly ordering and practicality. The main point is to see how hacking, making, and prototyping can go beyond the smart city visions and address real problems of the everyday life.

Even if initiatives as Uber and Airbnb might have started as social initiatives, the corresponding increase in popularity and economic benefits seemed to have encouraged the use of 'old economy' business models that continued dominating the further path. It is obvious that the current way of operation is far less 'addressing the common good', and that these initiatives no longer function as co-creative partnerships aiming for new city-making. Differently said, the value is no longer in and for the city. 
In our view, hackable city-making needs to become more pluralistic and needs to involve more (inter-)disciplinary stakeholders in a dialogical way in order to face today's challenges. Such participatory city-making process envisioning livable and sustainable urban environments goes far beyond simple, or even complex, product-service design; it has political, organizational, and even cultural implications. Consequently, a collaborative systemic approach emphasizing the human scale, is vital for engaging stakeholders from public sector, industry, education, and research as well as citizens in a shared process of knowledge production in which they collaboratively envision desired future cities (Brodersen et al. 2008; Carayannis and Campbell 2012; van Waart et al. 2016). It requires co-creative partnerships to actually team up towards a future direction. In this, the biggest challenge is to embrace a new collaborative attitude, a participatory approach, and to have a proper infrastructure that supports this social fabric. This new city-making process is not only about bringing various disciplines together that addresses urban developments, but foremost to establish a collaborative effort of defining a new way of working between professional designers, academics, policy-makers, and citizens.

Although co-creative partnerships seem to be the answer and time is ripe for a more collaborative approach, it is not straightforward that everybody can participate. However, the participatory turn and the maker movement have contributed largely to fact that 'everybody is a designer'. Making and participatory prototyping can empower citizens and allow them to express and to be part of the city-making process. Participatory city-making is about democracy, rather than politics; it is about caring together for a shared environment and making your own future. Interestingly, Barber (2013) clearly points out why mayors should rule the world; mayors have a city to manage, but also need the people to make their city. Hackable city-making, thus, can make a difference through involving emerging city-makers and by elaborating upon the interplay between systems thinking and design thinking.

Having the city as a patchable plug-in platform asks for participatory governance, a new paradigm in city-making, which combines top-down public management with bottom-up social innovation (e.g. Bria et al. 2014; Loorbach 2014; Mulder 2014). A patchable plug-in platform enables emerging city-makers to create value for the city, for society; however, it asks for new ways of city management too. New participatory ways of governance include advanced hacking, making, and prototyping skills, such as envisioning, translating, or orchestrating, and enable emerging, heterogeneous city-makers to participate actively in exploring the collaborative envisioned potential and constructive dialogue aiming for transformational change for the common good. 


\section{References}

Ampatzidou, Cristina, Matthijs Bouw, Froukje van de Klundert, Michiel de Lange, and Martijn de Waal. 2015. The Hackable City: A research manifesto and design toolkit. Amsterdam: Amsterdam Creative Industries Publishing.

Barber, Benjamin R. 2013. If Mayors ruled the world: dysfunctional nations, rising cities. New Haven, London: Yale University Press.

Bria, Francesca, Esteve Almirall, Peter Baeck, Harry Halpin, Jon Kingsbury, and Frank Kresin. 2014. Digital social innovation interim report. London: Nesta.

Brodersen, Christina, Christian Dindler, and Ole Sejer Iversen. 2008. Staging imaginative places for participatory prototyping. Co-Design 4 (1): 19-30.

Brown, Tim. 2008. Design thinking. Harvard Business Review 84-92.

Bureau of European Policy Advisors. 2010. Empowering people, driving change: Social innovation in the European Union. Luxembourg: Publications Office of the European Union.

Buxton, Bill. 2010. Sketching user experiences: Getting the design right and the right design. Burlington Morgan: Kaufmann.

Brynskov, Martin, Juan Carvajal Bermúdez, Manu Fernandez, Henrik Korsgaard, Ingrid Mulder, Katarzyna Piskorek, Lea Rekow, and Martijn de Waal. 2014. Urban interaction design: Towards city-making. Amsterdam: Floss Manuals.

Carayannis, Elias G., and David F.J. Campbell. 2012. Mode 3 knowledge production in quadruple helix innovation systems. New York: Springer.

CHIPS Magazine. 2002. Only the limits of our imagination. Exclusive interview with Rear Adm. Grace Hopper from Chips Ahoy July 1986. http://www.doncio.navy.mil/chips/ArticleDetails. aspx?id=3563. Accessed 30 Apr 2017.

de Waal, Martijn, Michiel de Lange, and Matthijs Bouw. 2017. The Hackable City: City-making in a platform society. Archit.

Jones, Peter. 2016. Proceedings of relating systems thinking and design (RSD5) 2016 symposium. Systemic Design. https://systemic-design.net/rsd-symposia/rsd5-2016/. Accessed 30 Apr 2017.

Junginger, Sabine. 2008. Product development as a vehicle for organizational change. Design Issues 24 (1): 26-35.

Karasti, Helena. 2014. Infrastructuring in participatory design. In Proceedings of PDC'14, the 13th participatory design conference, 141-150. New York: ACM.

Koopman, Philip, and Robert R. Hoffman. 2003. Work-arounds, make-work, and kludges. IEEE Intelligent Systems 18 (6): 70-75.

Kun, Peter, and Ingrid Mulder. 2016. Prototyping for citizen engagement. Workshop outcomes, design and the city. http://studiolab.ide.tudelft.nl/studiolab/transitiondesign/files/2016/ 07/prototyping-citizen-engagement-booklet-hq.pdf. Accessed 30 Apr 2017.

Levy, Steven. 2001. Hackers: Heroes of the computer revolution, vol. 4. New York: Penguin Books.

Loorbach, Derk. 2014. To transition! Governance panarchy in the new transformation. Inaugural address. Erasmus University Rotterdam.

Manzini, Enzio, and Francesca Rizzo. 2011. Small projects/large changes: Participatory design as an open participated process. CoDesign 7 (3-4): 199-215.

McPhee, Chris, Westerlund Mika, and Seppo Leminen. 2012. Editorial: living labs. Technology Innovation Management Review 2 (9): 3-5.

Morelli, Nicola, Ingrid Mulder, Grazia Concilio, Janice S. Pedersen, Tomasz Jaskiewicz, Amalia De Götzen, and Mark Aguilar. 2017. Open data as a new commons. Empowering citizens to make meaningful use of a new resource. In: Internet science. INSCI 2017, ed. Ioannis Kompatsiaris Jonathan Cave, Anna Satsiou, Georg Carle, Antonella Passani, Efstratios Kontopoulos, Sotiris Diplaris and Donald McMilan, 212-221. Switzerland: Springer International Publishing.

Mostert-van der Sar, Manon, Ingrid Mulder, Leo Remijn, and Peter Troxler. 2013. Fablabs in design education. In DS 76: Proceedings of E\&PDE 2013: International conference on engineering and product design education, 629-634. Dublin: Dublin University of Technology. 
Mulder, Ingrid. 2014. Sociable smart cities: Rethinking our future through co-creative partnerships. In DAPI 2014: Distributed, ambient, and pervasive interactions, vol. 8530, ed. Norbert Streitz, and Panos Markopoulos, 566-574. Lecture notes in computer science. Switzerland: Springer International Publishing.

Mulder, Ingrid. 2015. Opening up: Towards a sociable smart city. In Citizen's right to the digital city: Urban interfaces, activism, and placemaking, ed. Marcus Foth, Martin Brynskov, and Timo Ojala, 161-173. Singapore: Springer.

Mulder, Ingrid, and Derk Loorbach. 2018. Rethinking Design: A critical perspective to embrace societal challenges. In Can Design Catalyse the Great Transition: Papers from the Transition Design Symposium 2016, ed. Gideon Kossoff and Ruth Potts, 16-24, Dartington: United Kingdom.

Mulgan, Geoff. 2016. Digital social innovation: The programme and where next? Panel presentation at policy workshop shaping the future of digital social innovation in Europe, Brussels. https:// issuu.com/dsi4eu/docs/02_impact_04_geoff_mulgan_nesta.ppt. Accessed 30 Apr 2017.

Murray, Robin, Julie Calulier-Grice, and Geoff Mulgan. 2010. Open book of social innovation. The Young Foundation.

Rifkin, Jeremy. 2008. The third industrial revolution. Engineering and Technology 3 (7): 26-27.

Rittel, Horst W.J., and Melvin M. Webber. 1973. Dilemmas in a general theory of planning. Policy Sciences 4 (2): 155-169.

Sanders, Liz, and Pieter Jan Stappers. 2014. From designing to co-designing to collective dreaming: Three slices in time. Interactions 21 (6): 24-33.

Stafford-Fraser, Quentin. 2001. On site: The life and times of the first web cam. Communications of the ACM 44 (7): 25-26.

Suchman, Lucy A., Jeanette Blomberg, and Randal Trigg. 2002. Working artefacts: Ethnomethods of the prototype. British Journal of Sociology 53 (2): 163-179.

Thompson, Clive. 2005. Meet the life hackers. The New York Times Magazine. http://www.nytimes. com/2005/10/16/magazine/meet-the-life-hackers.html?_r=0. Accessed 28 Apr 2017.

Townsend, Anthony M. 2013. Smart cities: Big data, civic hackers, and the quest for a New Utopia. New York: WW Norton and Company.

Urban IxD Manifesto. 2014. http://urbanixd.eu/s/UrbanIxD_Manifesto.pdf. Accessed 28 Apr 2017. van Waart, Peter, Ingrid Mulder, and Cees de Bont. 2015. Participatory prototyping for future cities. In Proceedings of PIN-C 2015: Reframing design, the 4th participatory innovation conference, 337-343. The Hague: The Hague University of Applied Science.

van Waart, Peter, Ingrid Mulder, Cees de Bont. 2016. A participatory approach for envisioning a smart city. Social Sciences Computer Review 34 (6): 708-723.

Ingrid Mulder is an expert in design for social transformation, currently working as an Associate Professor at the Faculty of Industrial Design Engineering, Delft University of Technology. Thanks to her background in Policy and Organization Sciences (M.A., University of Tilburg) and Behavioural Sciences (Ph.D., University of Twente), her on-going research interestingly combines strategic design with diffuse design, and addresses the interplay between top-down policy and bottom-up participatory innovation. Her current projects have been awarded by prestigious grants: Open4Citizens (Horizon2020 CAPS) aims at empowering citizens to make meaningful use of open data; Participatory City-Making, funded by the NWO Research through Design call, considers the collaborative construction of new visions through small-scale experimenting as a way of triggering a process of broader change and transformation; and DESIGNSCAPES (Horizon2020 CO-CREATION) plays a fundamental role in building capacities to enhance design-enabled innovation in urban contexts. 
Péter Kun is an interaction designer with an interdisciplinary background, currently doing Ph.D. research at the Faculty of Industrial Design Engineering, Delft University of Technology. With his interdisciplinary background in Interaction Design and Technologies (M.Sc., Chalmers University of Technology), Industrial Management (B.Sc., Budapest University of Technology and Economics) and several years working as a workshop facilitator, in his research Péter investigates the intersection of design research and data science, and seeks new design techniques for exploratory and generative design, where data is used to fuel creativity, inform design concepts and be a source for inspiration. This research is conducted within the Open4Citizens (Horizon2020 CAPS) project, aimed at empowering citizens to make meaningful uses of open data.

Open Access This chapter is licensed under the terms of the Creative Commons Attribution 4.0 International License (http://creativecommons.org/licenses/by/4.0/), which permits use, sharing, adaptation, distribution and reproduction in any medium or format, as long as you give appropriate credit to the original author(s) and the source, provide a link to the Creative Commons license and indicate if changes were made.

The images or other third party material in this chapter are included in the chapter's Creative Commons license, unless indicated otherwise in a credit line to the material. If material is not included in the chapter's Creative Commons license and your intended use is not permitted by statutory regulation or exceeds the permitted use, you will need to obtain permission directly from the copyright holder. 